\title{
Telomere dynamics in the first year of life, but not later in life, predict lifespan in a wild bird
}

\author{
Elizabeth Sheldon ${ }^{1}$, Justin Eastwood ${ }^{2}$, Niki Teunissen ${ }^{2}$, Michael Roast ${ }^{2}$, Marie Fan ${ }^{2}$, \\ Michelle Hall ${ }^{3}$, Nataly Hidalgo Aranzamendi ${ }^{2}$, Sjouke Kingma ${ }^{4}$, Simon Verhulst ${ }^{5}$, and \\ Anne Peters ${ }^{2}$ \\ ${ }^{1}$ University of South Florida \\ ${ }^{2}$ Monash University \\ ${ }^{3}$ University of Melbourne \\ ${ }^{4}$ Max-Planck-Institute for Ornithology \\ ${ }^{5}$ University of Groningen
}

May 22, 2021

\begin{abstract}
Telomeres are protective, nucleoprotein structures at the end of chromosomes that have been associated with lifespan across taxa. However, the extent to which these associations can be attributed to absolute length versus the rate of telomere shortening prior to sampling remains unresolved. In a longitudinal study, we examined the relationship between lifespan, telomere length and the rate of telomere shortening in wild, purple-crowned fairy-wrens (Malurus coronatus coronatus). To this end, we measured telomere length using qPCR in the blood of 59 individuals sampled as nestling and 4-14 months thereafter, and in 150 individuals sampled on average three times across adulthood. We applied within-subject centering analyses to simultaneously test for associations between lifespan and average telomere length and telomere shortening. We reveal that the rate of telomere shortening in the first year of life predicted lifespan, with individuals with faster shortening rates living less long. We also report a trend for an effect of telomere length in the first year of life on lifespan, independent of telomere shortening. In contrast, in adulthood neither telomere shortening, nor telomere length predicted lifespan, despite a considerably larger data set. Our results suggest that telomere length measured very early in life (during development) and longitudinal assessments of telomere shortening during the first year of life constitute more useful biomarkers of total life expectancy than either telomere length measured after development, or telomere shortening later in adulthood.
\end{abstract}

\section{Hosted file}

Early life telomere changes predict lifespan.docx available at https://authorea.com/users/ 404965/articles/523041-telomere-dynamics-in-the-first-year-of-life-but-not-later-inlife-predict-lifespan-in-a-wild-bird 
a)

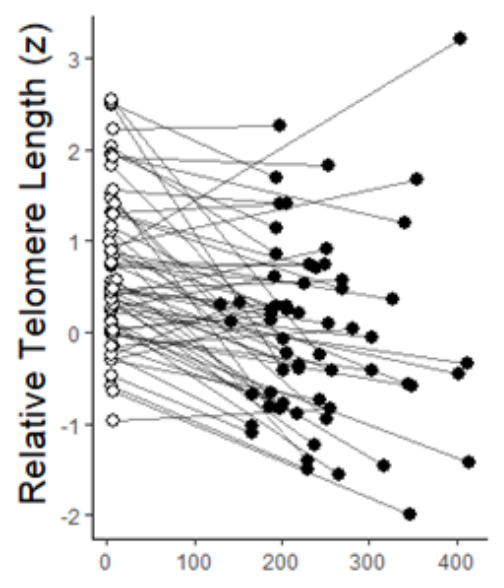

b)
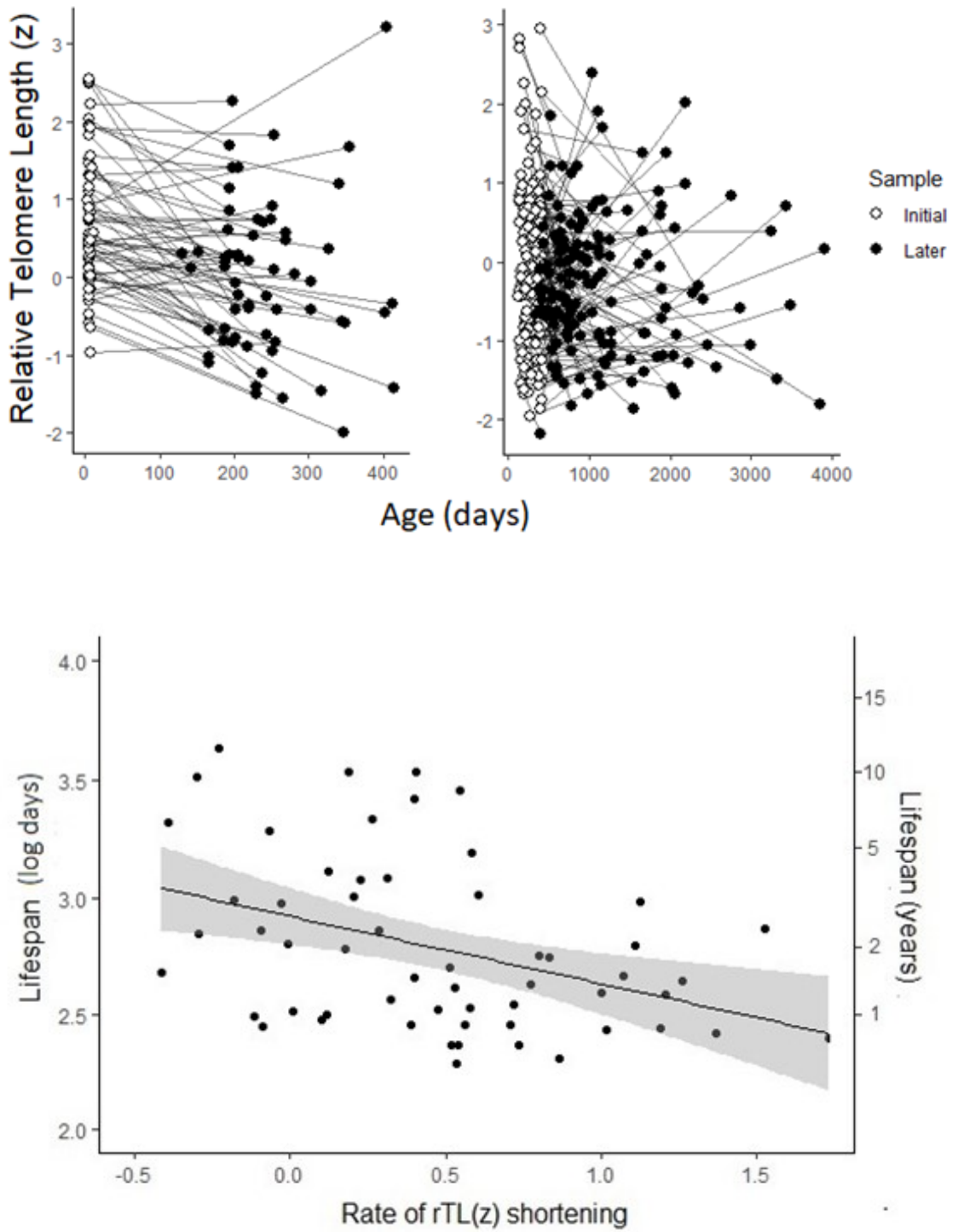


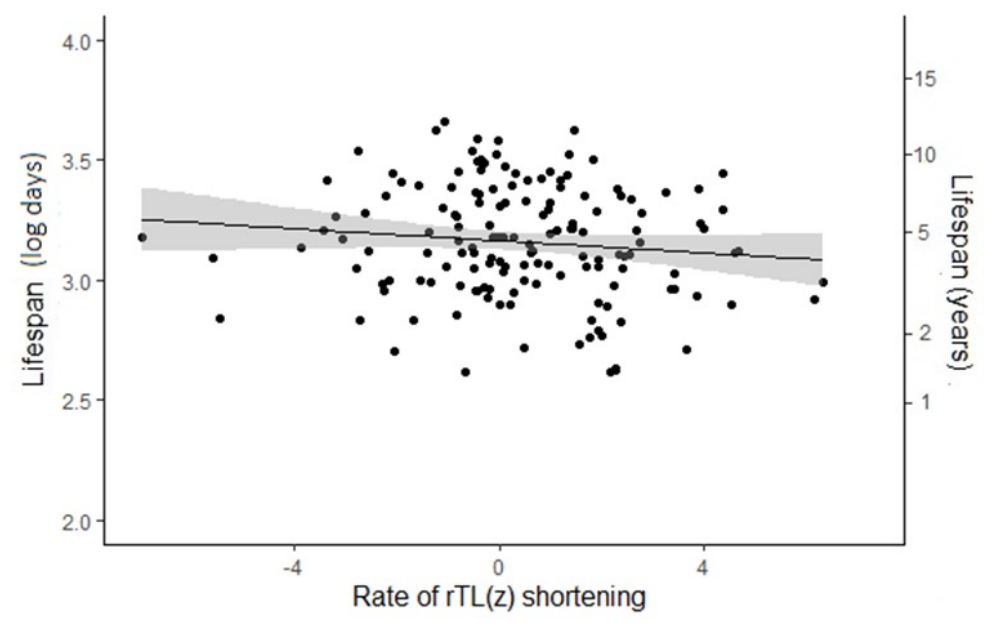

